\title{
Particle Finite Element Simulation of Chip Formation in Cutting Processes
}

\author{
Matthias Sabel ${ }^{1, a}$, Christian Sator ${ }^{1}$, Ralf Müller ${ }^{1, b}$, and Benjamin Kirsch ${ }^{2}$ \\ ${ }^{1}$ Institute of Applied Mechanics, University of Kaiserslautern, Gottlieb-Daimler-Str., \\ D-67663 Kaiserslautern
}

${ }^{2}$ Institute for Manufacturing Technology and Production Systems, University of Kaiserslautern, Gottlieb-Daimler-Str., D-67663 Kaiserslautern

amsabel@rhrk.uni-kl.de, bram@rhrk.uni-kl.de

Keywords: PFEM, alpha-shape, cutting simulations

\begin{abstract}
The formation of chips in cutting processes is characterised by large deformations and large configurational changes and therefore challenges established modeling techniques. To overcome these difficulties, the particle finite element method (PFEM) combines the benefits of discrete modeling techniques with methods based on continuum mechanics. In this work an outline of the PFEM, as well as an explanation of the finite element formulation are provided. The impact of the boundary detection on the structural integrity is studied. The numerical examples include a tensile test as well as cutting simulations. The paper is concluded by a comparison of cutting forces with analytical results.
\end{abstract}

\section{Introduction}

The particle finite element method (PFEM) was first applied to problems with sloshing liquids in [1,2] where large changes in the topology overexert the standard finite element method. The ability to cope with these large topological changes designates the PFEM as a convenient tool for the simulation of cutting processes, where the chip formation challenges the standard finite element method (FEM). Approaches to model the cutting of metals can be found in $[3,4,5]$. Since the material modeling in the PFEM is based on continuum mechanics, length scales of a workpiece can be considered, which is critical in discrete methods. In order to model the cutting of metals, plasticity at large deformations is considered in the material model. The PFEM algorithm consist of several steps, which are illustrated in Fig. 1.

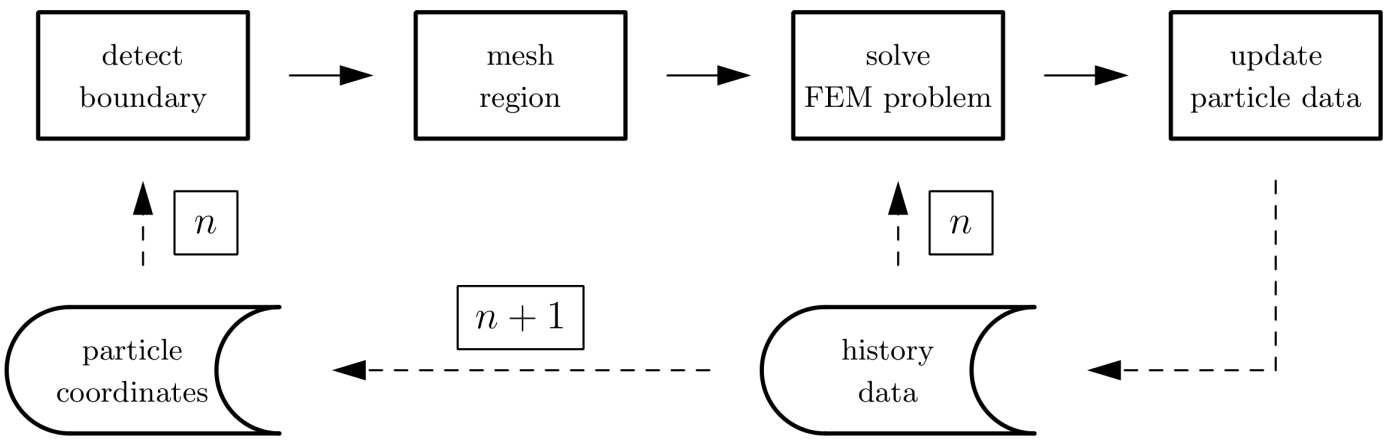

Fig. 1: Scheme of the PFEM algorithm

The PFEM algorithm described in this work starts with the processing of a set of particles. To form a domain and later a finite element mesh, the boundary needs to be detected. This process is realised with the so called $\alpha$-shape algorithm, which was first used in the field of computer graphics [9] and has been adapted to the PFEM in [1]. For every pair of particles a circle is defined that intersects both points. If there are no other particles located within this circle, the two intersecting particles form a 
boundary segment. The radius of the $\alpha$-circle is defined as $r=\alpha H$, where $H$ is the smallest distance between any two particles in the undeformed set. After identifying the boundary, the domain is meshed with the program Triangle [7]. In conjunction with boundary conditions a finite element problem can now be solved with the finite element (FE) programme Feap [8]. The resulting displacements form the FE simulation are used to update the particle coordinates, and the current deformation gradient is stored as history data for the following PFEM loading step.

\section{Physical Interpretation of the $\alpha$-Shape Method}

The $\alpha$-shape method was originally developed in the field of computer graphics to detect the shape of a set of particles, and its central parameter $\alpha$ controls the level of detail of the detected shape. In $[1,2]$ the $\alpha$-shape method is adopted in PFEM simulations of sloshing liquids, and recommended values for the parameter $\alpha$ are presented based on observations. However, PFEM applications in solid mechanics reveal an influence of $\alpha$ on the material response and the structural integrity. In order to find a physical interpretation for the parameter $\alpha$, the effect of the $\alpha$-shape method on the boundary of a continuous body during deformation is studied. At this point the authors want to mention that the following considerations are only valid for spatially uniform deformations. However, the outcome of this analysis is valuable and can provide a better understanding of the role of the $\alpha$-shape algorithm in PFEM simulations. In Fig. 2, we consider a body in its reference configuration $\mathcal{B}_{0}$. The boundary of the body is occupied by a set of particles $S^{b}$ which are distributed in equal distances and therefore form boundary segments of equal length. Furthermore, we assume that the boundary in the undeformed configuration is established and that $\mathrm{d} L$ is the length of the boundary segments in this configuration. We define the orientation of the boundary segments in configuration $\mathcal{B}_{0}$ by the unit vector $\boldsymbol{H}$. In Fig. 2, the undeformed- and the deformed configuration $\mathcal{B}_{t}$ are shown as well as the line segment $\boldsymbol{H}$ ( $\boldsymbol{h}$ resp.) and the $\alpha$-circles.

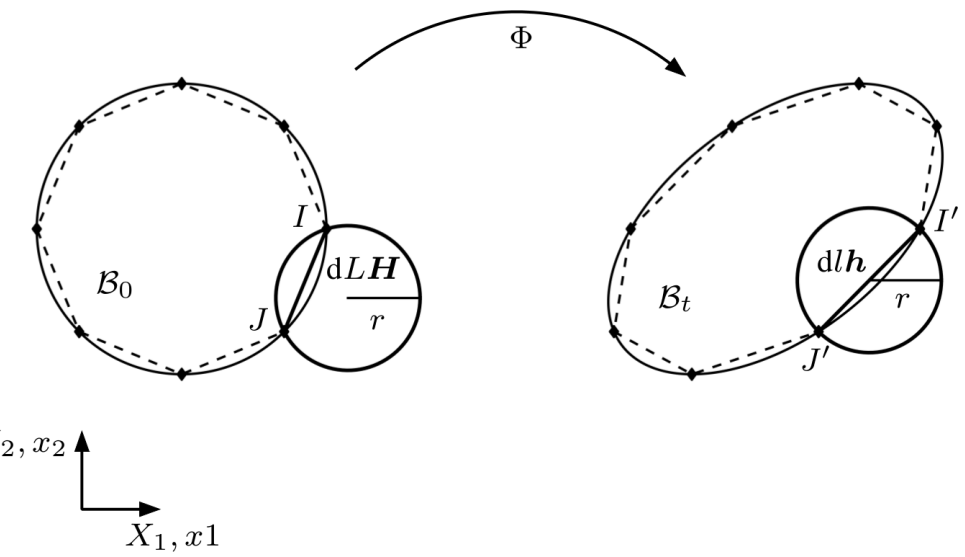

Fig. 2: Boundary of $\mathcal{B}$ with $\alpha$-circles in reference- and spatial-configuration

The segment in the reference configuration is represented by $\mathrm{d} L \boldsymbol{H}$ which points from particle $J$ to particle $I$. Particle $J$ occupies the position $\boldsymbol{X}_{J}$ and $I$ is therefore located at $\boldsymbol{X}_{I}=\boldsymbol{X}_{J}+\mathrm{d} L \boldsymbol{H}$. Then the line segment in the deformed configuration $\mathrm{d} l \boldsymbol{h}$ is defined by the particles $J^{\prime}$ and $I^{\prime}$. The particle $J^{\prime}$ is at position $\boldsymbol{x}_{J}$ and $I^{\prime}$ is located at $\boldsymbol{x}_{I}=\boldsymbol{x}_{J}+\mathrm{d} l \boldsymbol{h}$. The location of particle $J^{\prime}$ can also be defined using the deformation $\Phi$ as

$$
\boldsymbol{x}_{J}=\Phi\left(\boldsymbol{X}_{J}\right)
$$

and similarly for $I^{\prime}$

$$
\boldsymbol{x}_{I}=\boldsymbol{x}_{J}+\mathrm{d} l \boldsymbol{h}=\Phi\left(\boldsymbol{X}_{J}+\mathrm{d} L \boldsymbol{H}\right)
$$


Another definition of $\boldsymbol{x}_{I}$ can be defined by using a Taylor expansion. Here, only the first order term of the expansion is regarded which yields

$$
\boldsymbol{x}_{I}=\boldsymbol{x}_{J}+\mathrm{d} l \boldsymbol{h}=\boldsymbol{x}_{J}+\frac{\partial \Phi\left(\boldsymbol{X}_{J}\right)}{\partial \boldsymbol{X}_{J}} \mathrm{~d} L \boldsymbol{H}
$$

where $\boldsymbol{F}=\partial \Phi\left(\boldsymbol{X}_{J}\right) / \partial \boldsymbol{X}_{J}$ represents the deformation gradient. Using this notation we can express Eq. (3) as

$$
\mathrm{d} l \boldsymbol{h}=\boldsymbol{F} \mathrm{d} L \boldsymbol{H},
$$

which leads to the definition of the stretch ratio

$$
\lambda=\frac{\mathrm{d} l}{\mathrm{~d} L}=\boldsymbol{h}^{T} \boldsymbol{F} \boldsymbol{H} .
$$

By squaring Eq. (4) on both sides, and rearranging in terms of $\lambda^{2}=(\mathrm{d} l / \mathrm{d} L)^{2}$ we can express the quadratic stretch

$$
\lambda^{2}=\boldsymbol{H}^{T} \boldsymbol{C H},
$$

where $\boldsymbol{C}=\boldsymbol{F}^{T} \boldsymbol{F}$ represents the right Cauchy-Green tensor. In order to demonstrate the influence of the parameter $\alpha$ on the boundary during a deformation, we have to recall the working principle of the $\alpha$-shape method. Within the algorithm a circle is defined for each pair $I$ and $J$ of particles, where the radius is scaled by $\alpha$. This leads to the conclusion that the distance between the respective pair of particles must not exceed the diameter of the $\alpha$-circle. In the example presented in Fig. 2, the radius of the circle is defined as $r=\alpha \mathrm{d} L$, and therefore the length of the deformed segment needs to fulfil the condition

$$
\mathrm{d} l \leq 2 \alpha \mathrm{d} L .
$$

Inserting Eq. (7) into Eq. (5) we can define a critical stretch ratio

$$
\lambda_{c}=2 \alpha,
$$

and recalling the quadratic stretch from Eq. (6) the following quadratic form can be defined

$$
4 \alpha^{2} \geq \boldsymbol{H}^{T} \boldsymbol{C H}
$$

In order to present a relation between $\alpha$ and the eigenvalues of the right Cauchy-Green tensor, we rearrange Eq. (6) to

$$
\mathbf{0}=\left(\boldsymbol{C}-\mathbf{1} \lambda^{2}\right) \boldsymbol{H}
$$

Eq. (10) represents a common eigenvalue problem and it can be observed that the undeformed line segment $\boldsymbol{H}$ has to be collinear to the eigenvector corresponding to $\lambda_{1 / 2}$ in order to fulfil Eq. (10). In [6] a more detailed description on the topic is provided as well as illustrative examples.

\section{Finite Element Formulation}

Within the PFEM a formulation in the current configuration is very suitable. Thus the equilibrium condition is given in an Eulerian setting by

$$
\operatorname{div} \boldsymbol{\sigma}+\boldsymbol{b}=\mathbf{0}
$$


where $\boldsymbol{\sigma}$ is the Cauchy stress tensor, and the vector $\boldsymbol{b}$ represents a volume force. As can be seen in Eq. (11), we focus on the static problem in this work. The weak form can then be derived by multiplying Eq. (11) with a test function $\boldsymbol{\eta}$ and applying the Cauchy theorem. The weak form then yields

$$
\int_{\mathcal{B}_{t}} \boldsymbol{\sigma} \cdot \operatorname{grad} \boldsymbol{\eta} d \Omega-\int_{\mathcal{B}_{t}} \boldsymbol{b} \cdot \boldsymbol{\eta} d \Omega-\int_{\partial \mathcal{B}_{t}^{\sigma}} \boldsymbol{t} \cdot \boldsymbol{\eta} d \Gamma=0 .
$$

Since Eq. (12) is nonlinear in the geometry and - as will be demonstrated later - nonlinear in the material, the equation is linearised and solved using a Newton scheme. The spatial discretisation of the linearised problem is realised with triangular finite elements, which are characterised by the shape functions

$$
N_{1}(r, s)=1-r-s, N_{2}(r, s)=r, N_{3}(r, s)=s .
$$

The shape functions in Eq. (13) are defined using the coordinates $r$ and $s$ of an isoparametric reference element. Within the isoparametric concept the same shape functions are used to approximate the geometry as well as the unknowns. For more information on the isoparametric concept, the reader is referred to [17]. The shape functions are also used to transfer history data, which is stored at the particles to the Gauß points. This procedure has to be carried out for the deformation gradient $\boldsymbol{F}$ in every time step of the finite element simulation. Only in the first Newton iteration of the first time step, the same procedure has to be carried out for the plastic deformation gradient $\boldsymbol{F}^{\mathrm{p}}$ and the hardening variable $\psi$. When the element routine is called, the previous gradients at the Gauß point level can then be computed with

$$
\boldsymbol{F}_{\mathrm{GP}, \mathrm{old}}=\sum_{I=1}^{3} N_{I}\left(r^{\mathrm{GP}}, s^{\mathrm{GP}}\right) \boldsymbol{F}_{I, \mathrm{old}}, \quad \boldsymbol{F}_{\mathrm{GP}, \mathrm{old}}^{\mathrm{p}}=\sum_{I=1}^{3} N_{I}\left(r^{\mathrm{GP}}, s^{\mathrm{GP}}\right) \boldsymbol{F}_{I, \text { old }}^{\mathrm{p}}
$$

and the previous hardening variable at the element level is defined as

$$
\psi_{\mathrm{GP}, \mathrm{old}}=\sum_{I=1}^{3} N_{I}\left(r^{\mathrm{GP}}, s^{\mathrm{GP}}\right) \psi_{I, \mathrm{old}} .
$$

The total deformation gradient can then be computed using the multiplicative decomposition

$$
\boldsymbol{F}=\boldsymbol{F}_{\text {act }} \boldsymbol{F}_{\mathrm{GP}, \text { old }} .
$$

In Eq. (16) $\boldsymbol{F}^{\text {act }}$ represents the deformation gradient of the actual PFEM load step, where $\boldsymbol{F}$ defines the gradient of the total deformation i.e. all PFEM load steps. The incrementation in load steps is necessary to ensure the boundary detection and meshing. The multiplicative decomposition in Eq. (16) was first mentioned in [20] in the context of elasto plasticity to split the deformation gradient in an elastic, and plastic part

$$
\boldsymbol{F}=\boldsymbol{F}^{\mathrm{e}} \boldsymbol{F}^{\mathrm{p}}
$$

This decomposition is applied in this work as well, since we want to model plasticity at finite strains. In order to compute the plastic deformation gradient, an extension of the return mapping algorithm to finite deformations is applied as in [14]. To extend the model from small strains to finite deformations the Hencky strain is introduced

$$
\varepsilon=\frac{1}{2} \ln (C)
$$


for more details the reader is referred to [14]. In Eq. (18) $\boldsymbol{C}=\boldsymbol{F}^{\mathrm{T}} \boldsymbol{F}$ is the right Cauchy-Green tensor. The stress update algorithm for small strains is called with the elastic trial strain in the Hencky setting, according to

$$
\varepsilon_{\mathrm{tr}}^{\mathrm{e}}=\frac{1}{2} \ln \left(C_{\mathrm{tr}}^{\mathrm{e}}\right)
$$

with the trial strain

$$
\boldsymbol{C}_{\mathrm{tr}}^{\mathrm{e}}=\left(\boldsymbol{F}_{\mathrm{tr}}^{\mathrm{e}}\right)^{T} \boldsymbol{F}_{\mathrm{tr}}^{\mathrm{e}}
$$

and

$$
\boldsymbol{F}_{\mathrm{tr}}^{\mathrm{e}}=\boldsymbol{F}\left(\boldsymbol{F}_{\mathrm{n}}^{\mathrm{p}}\right)^{-1}
$$

In Eq. (21) and (23) the index $\mathrm{n}$ denotes the previous time step of the stress update algorithm i.e. the previous Newton iteration. The current time step $n+1$ is omitted in Eq. $19-21$ in order not to overload the notation. It is important to note that a PFEM simulation consists of several loading steps that follow a remeshing procedure. In each PFEM step, a FEM problem is solved, where the load can be incremented as well to ensure good convergence in the Newton's method. To model strain hardening, the following power law is considered

$$
\sigma_{y}=\sigma_{i y}+k \psi^{m}
$$

where $\sigma_{y}$ represents a yield stress depending on the hardening variable $\psi$. The initial yield stress $\sigma_{i y}$, the hardening modulus $k$, and the hardening exponent $m$ are constitutive parameters. Since Eq. (22) is nonlinear in the hardening variable and the hardening variable is updated by an evolution law depending on the plastic multiplier $\Delta \gamma$, a Newtons method is applied on the element level to determine $\Delta \gamma$ and $\psi$ respectively, for details see for example [18]. The small strain stress update algorithm provides the plastic small strain increment $\Delta \varepsilon^{\mathrm{p}}$ and the updated hardening variables. The discretisation in time leads to the update rule for the plastic deformation gradient

$$
\boldsymbol{F}_{\mathrm{n}+1}^{\mathrm{p}}=\exp \left(\Delta \varepsilon^{\mathrm{p}}\right) \boldsymbol{F}_{\mathrm{n}}^{\mathrm{p}}
$$

With this relation, the plastic deformation gradient can be recovered from the small plastic strain increment. Now the element residual and the element tangential stiffness matrix can be formed

$$
\overline{\boldsymbol{r}}_{I}^{e}=\int_{\Omega_{e}} \overline{\boldsymbol{B}}_{I}^{T} \boldsymbol{\sigma} \mathrm{d} \Omega, \quad \overline{\boldsymbol{k}}_{I J}^{e}=\int_{\Omega_{e}} g_{I J} 1 \mathrm{~d} \Omega+\int_{\Omega_{e}} \overline{\boldsymbol{B}}_{I}^{T} \mathbb{C} \overline{\boldsymbol{B}}_{J} \mathrm{~d} \Omega,
$$

where $\mathbb{C}$ represents the material tangent and $g_{I J}$ is the geometric stiffness. The global residual and tangential stiffness matrix follow by the assembly

$$
\overline{\boldsymbol{r}}=\bigcup_{e=1}^{n e} \overline{\boldsymbol{r}}^{e}, \quad \overline{\boldsymbol{k}}=\bigcup_{e=1}^{n e} \overline{\boldsymbol{k}}^{e}
$$

and the linear system $\overline{\boldsymbol{k}} \Delta \overline{\boldsymbol{u}}=\overline{\boldsymbol{r}}$ can be solved for $\Delta \overline{\boldsymbol{u}}$. Finally, the displacement is updated with $\overline{\boldsymbol{u}}_{n+1}=\overline{\boldsymbol{u}}_{n}+\Delta \overline{\boldsymbol{u}}$. For more elaborate details, such as the algorithmic tangent, the reader is referred to $[15,14,16]$. To determine the plastic strain increment $\Delta \varepsilon^{p}$, a stress update algorithm known from small strain theory can be used (see $[18,19,17])$. In order to store the history data $\left(\boldsymbol{F}, \boldsymbol{F}^{p}\right.$, and $\left.\psi\right)$ have to be transferred to the FE nodes. Therefore, a linear system of equations of the form

$$
\sum_{J=1}^{N} M^{I J} \boldsymbol{F}^{J}=P_{I}\left(\boldsymbol{F}^{\mathrm{GP}}\right)
$$


can be assembled, where

$$
\begin{gathered}
M_{I J}=\int_{\mathcal{B}_{t}} N_{I}\left(r^{\mathrm{GP}}, s^{\mathrm{GP}}\right) N_{J}\left(r^{\mathrm{GP}}, s^{\mathrm{GP}}\right) d \Omega \quad \text { and } \\
P_{I}\left(\boldsymbol{F}^{\mathrm{GP}}\right)=\int_{\mathcal{B}_{t}} N_{I}\left(r^{\mathrm{GP}}, s^{\mathrm{GP}}\right) \boldsymbol{F}^{\mathrm{GP}} d \Omega .
\end{gathered}
$$

In order to avoid the solution of (26) a lumping technique is used. Therefore the linear system (26) becomes a diagonal system

$$
\bar{M}^{I I} \boldsymbol{F}^{I}=P_{I}\left(\boldsymbol{F}^{\mathrm{GP}}\right)
$$

with the lumped system matrix

$$
\bar{M}_{I I}=\sum_{J=1}^{N} M_{I J}=\int_{\mathcal{B}_{t}} N_{I}\left(r^{\mathrm{GP}}, s^{\mathrm{GP}}\right) d \Omega
$$

After this projection to the FE nodes, the deformation gradients are stored as particle data in the PFEM method. In the next time step the deformation gradients are available as $\boldsymbol{F}^{\text {old }}$ at the Gauß point by an approximation as in (14). This procedure has to be performed not only for the deformation gradient $\boldsymbol{F}$, but for the plastic deformation gradient $\boldsymbol{F}^{p}$ as well as for the hardening variable $\psi$. It should be noted here that $\boldsymbol{F}^{p}$ and $\psi$ are only included in the first Newton iteration of the first time step, since these variables are iteratively determined in the Newton scheme.

\section{Tensile Tests on Elasto Plastic Material}

In the preceding sections it was pointed out that $\alpha$ can be regarded as the maximum stretch of a boundary segment. If the critical value $\lambda=2 \alpha$ is exceeded, the material separates. In order to demonstrate the meaning of $\alpha$ on an engineering based example, a tensile test is simulated. Fig. 3 shows the undeformed and the deformed specimen.

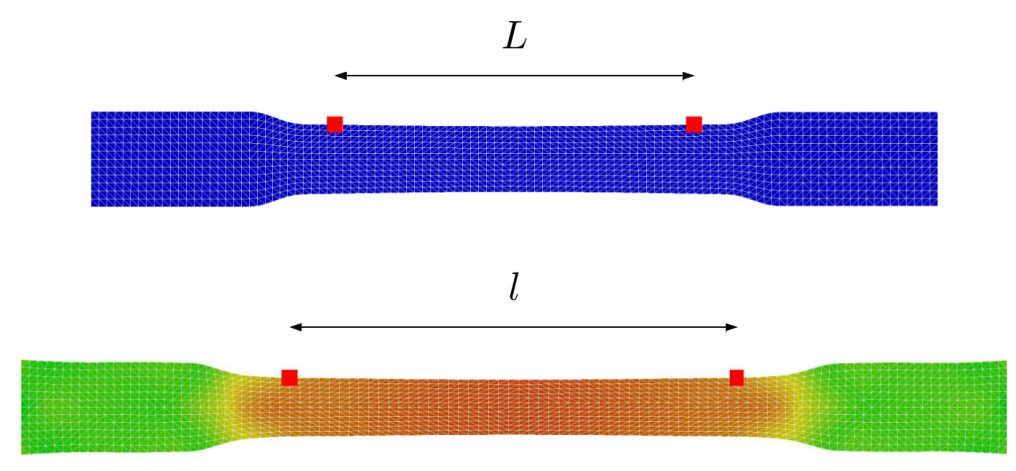

Fig. 3: Test specimen with undeformed and deformed length of midsection

In Fig. $3 L$ represents the reference length and $l$ denotes the deformed length. The undeformed overall length of the specimen is $160 \mathrm{~mm}$. In order to study the influence of $\alpha$ on the material response the reaction force is plot with respect to the stretch of the midsection $l / L$. For all simulations, the Young's modulus is set to $E=210000 \mathrm{MPa}$, the Poisson's ratio $\nu=0.3$, the hardening modulus $k=1600 \mathrm{MPa}$, the initial yield stress $\sigma_{i y}=300 \mathrm{MPa}$, and the exponent of the power law $m=0.8$. In Fig. 4 the reaction forces are plot with respect to the stretch of the midsection for three different values of $\alpha$. 


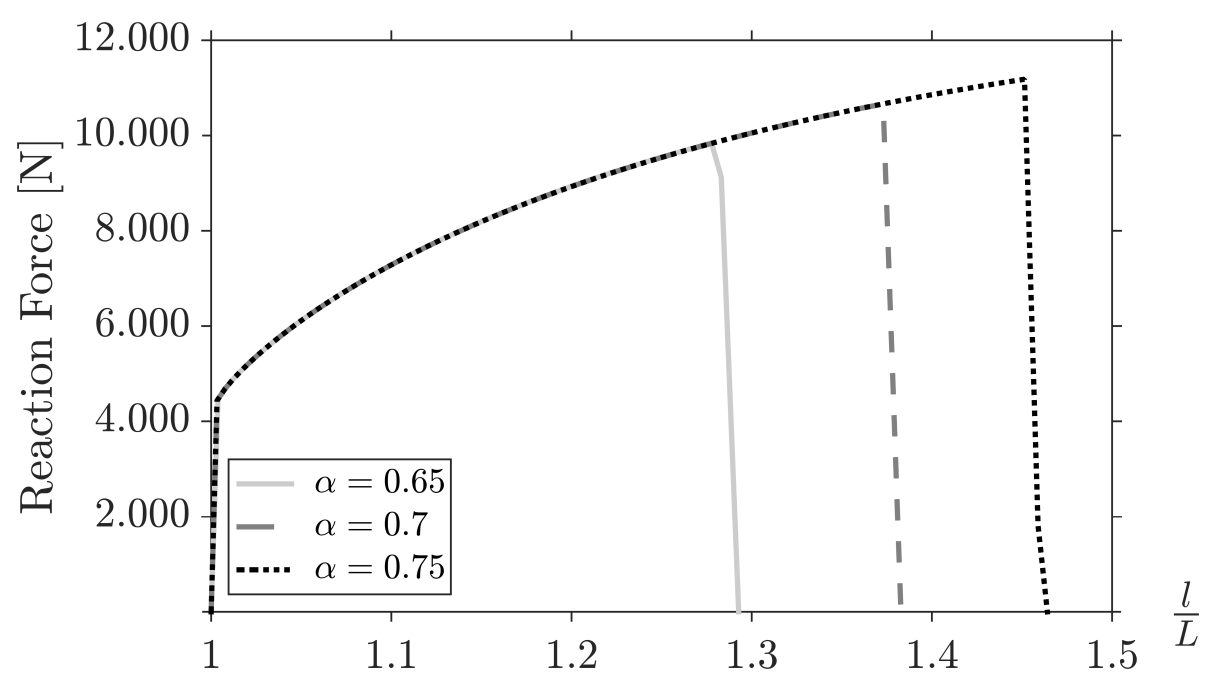

Fig. 4: Material response for values of $\alpha$

As can be seen in Fig. 4 increasing values of $\alpha$ lead to increasing stretches before the specimen separates.

\section{Cutting Simulations}

In the following simulations the tool is regarded as rigid body and only the workpiece is considered as deformable. The workpiece is driven horizontally towards the fixed tool, where - for simplicity only - normal contact is considered, i.e. orthogonal cutting is modeled. In this section the results from a simulation where the Young's modulus $E=210000 \mathrm{MPa}$, the Poisson's ratio $\nu=0.3$, the hardening modulus $k=E / 100$, the initial yield stress $\sigma_{i y}=235 \mathrm{MPa}$, and the exponent of the power law $m=1.0$ are presented. The depth of cut is set to $0.5 \mathrm{~mm}$. Fig. 5, 6, 7, and 8 illustrate stress and deformation patterns as described in classical textbooks on manufacturing and cutting like [21,22].

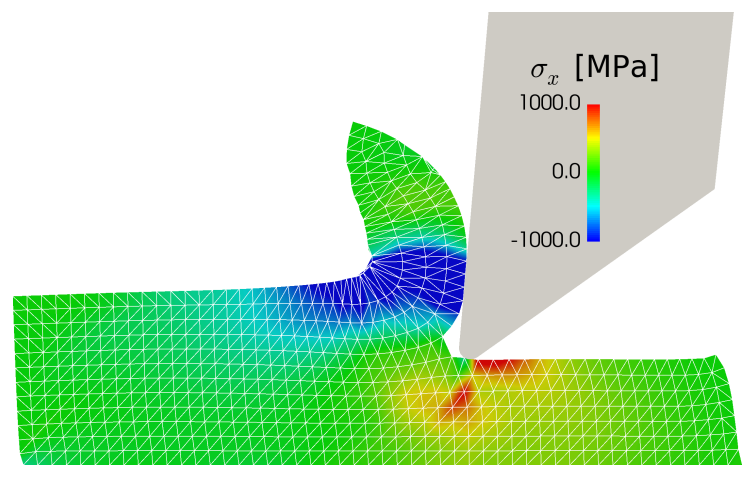

Fig. 5: $\sigma_{11}$ stress in cutting process

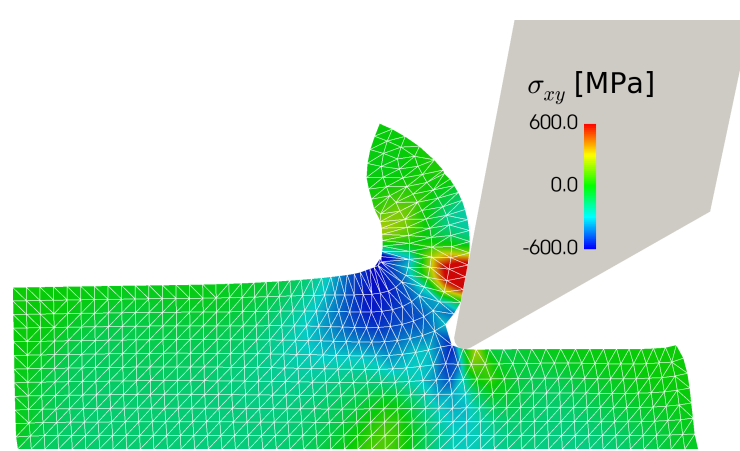

Fig. 6: Primary and secondary shear zone

Fig. 5 presents a region of pressure stresses in front of the cutting tool, and tensile stresses underneath the tool, where the material is stretched due to the chip formation. In Fig. 6 two regions of high shear stresses can be observed. The region with shear stresses of a negative sign is often called primary shear zone, whereas the region with positive shear stresses is referred to as secondary shear zone at the rake face (see [21, 22]). Fig. 7 and 8 show a contour plot of the norm of the plastic deformation gradient during cutting. 


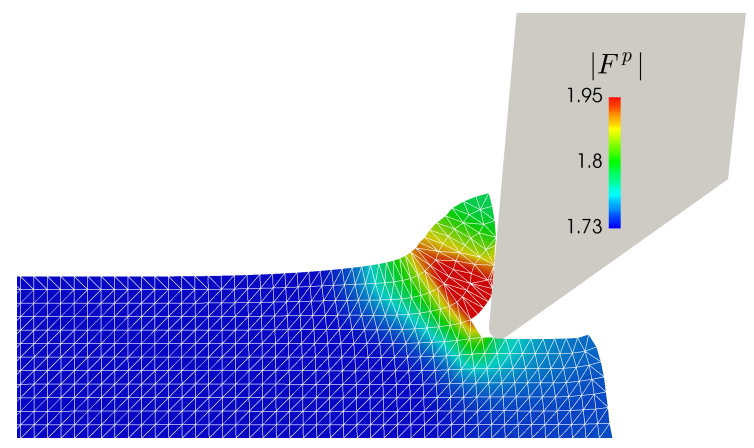

Fig. 7: Plastic deformation

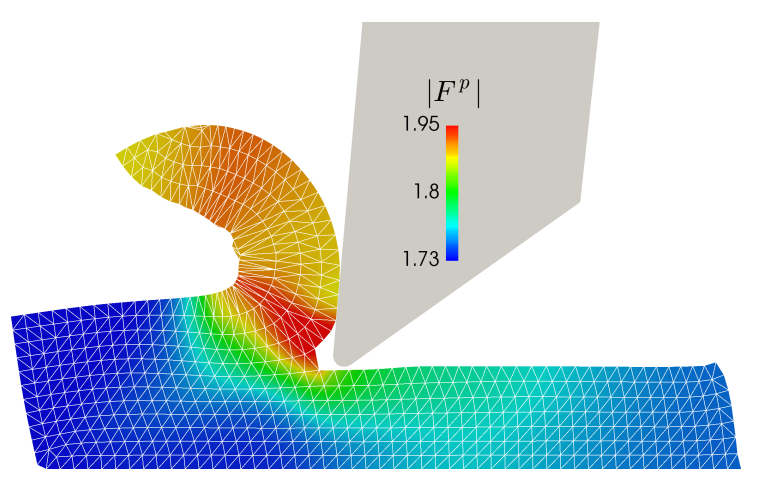

Fig. 8: Plastic deformation in progressed cut

The plastic deformation as shown in Fig. 7 and 8 intensifies with the progression of the cutting. In Fig. 7 a small chip has formed and strong plastic deformations can be observed in the primary shear zone (see Fig. 6). In Fig. 8 the cut has progressed and the chip shows large plastic deformations. Here, the workpiece exhibits small plastic deformations in the whole segment behind the cutting tool, i.e. the machined surface.

\section{Parameter Study on $\alpha$}

Since we have established that the value of $\alpha$ affects the material separation, a parameter study on $\alpha$ is carried out in this section. The cut in the workpiece progresses due to the failure of boundary segments in front of the cutting tool, i.e. the rake face. Fig. 9 and 10 present the results of cutting simulations for two values of $\alpha$.

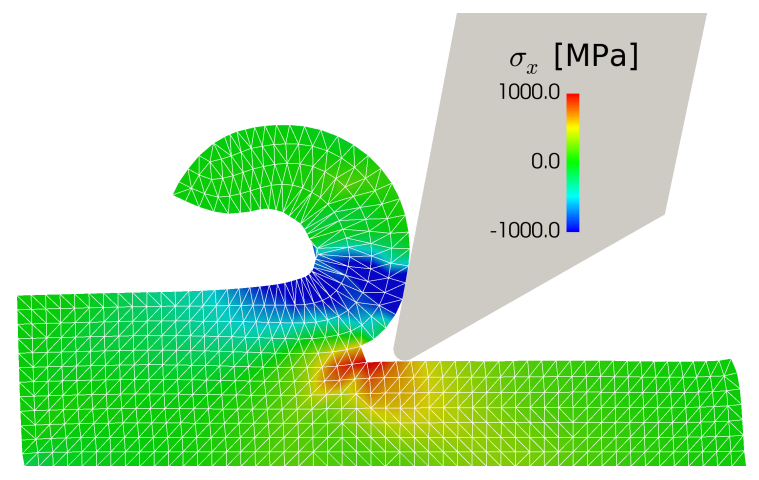

Fig. 9: Stress $\sigma_{x}$ for $\alpha=0.7$

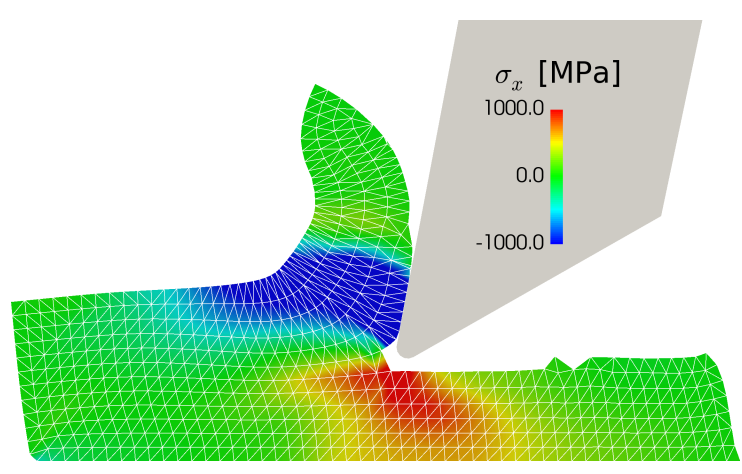

Fig. 10: Stress $\sigma_{x}$ for $\alpha=0.9$

Fig. 9 shows the result of a cutting simulation with $\alpha=0.7$. The low value of $\alpha$ causes an early separation, which mimics a rather brittle material. In comparison to Fig. 10 the tensile stresses underneath the cutting tool are reduced and the zone of material separation advances in a certain distance in front of the rake face. In Fig. 10 the material separates immediately in front of the tool and the material shows a more ductile behavior. The higher value of $\alpha$ leads to a large region of tensile stresses, and the chip thickness is increased. Fig. 11 shows a plot of the cutting forces for a variation of $\alpha$. 


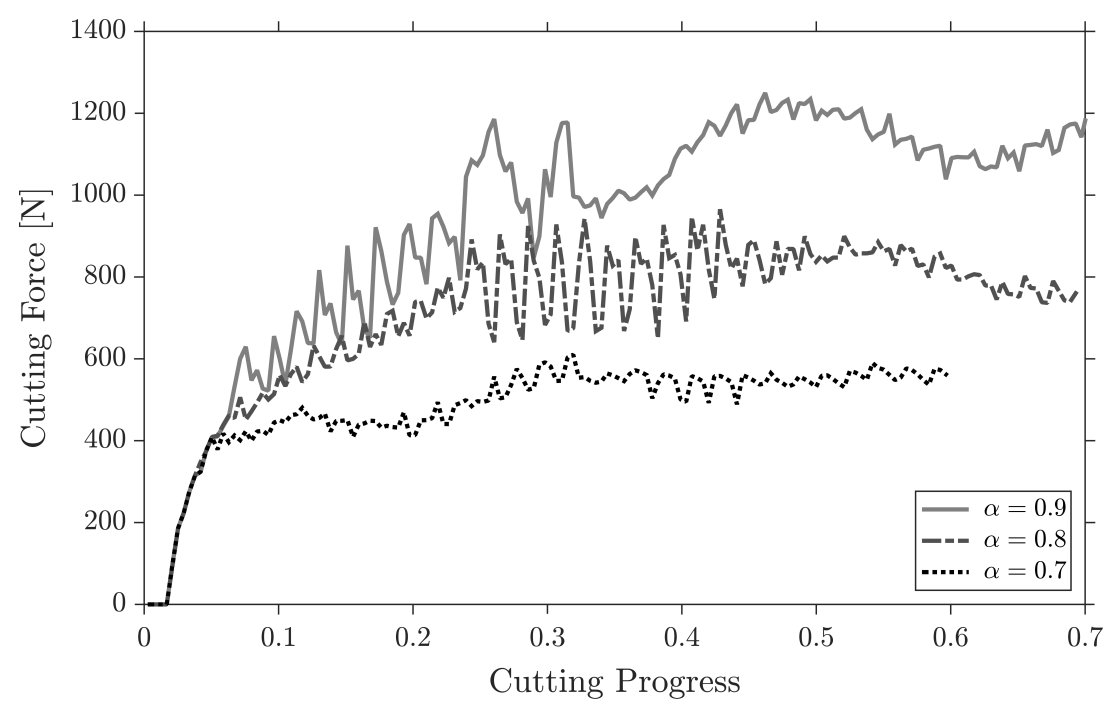

Fig. 11: Cutting forces for varying value of $\alpha$

The graphs in Fig. 11 present the cutting force plot with respect to the cutting progress $\bar{u} / L$, where $\bar{u}$ represents the penetration depth of the cutting tool and $L$ ist the length of the workpiece. At the start of the cutting process the cutting forces seem to coincide. However, at a certain point they diverge due to the different values of $\alpha$. The simulation with $\alpha=0.9$ allows the highest stretch before the material separation and therefore the value of the cutting force increases the most. The cutting force of the simulation with $\alpha=0.7$ is significantly lower, since the line segments in front of the cutting tool fail at a much lower stretch.

\section{Comparison to Analytical Results}

In manufacturing technology a concept has been developed in [23] to model the required power in turning processes. In order to calculate the power, the cutting force is calculated, which provides an analytical reference for the cutting force computations with the PFEM. The resultant force $\boldsymbol{F}_{z}$ is a product of the cutting process and in general is a force in three dimensional space. This resultant force consists of the components

$$
\boldsymbol{F}_{z}=\boldsymbol{F}_{c}+\boldsymbol{F}_{f}+\boldsymbol{F}_{p}
$$

In Eq. (31) $\boldsymbol{F}_{c}$ represents the cutting force, which acts in tangential direction of the turning workpiece. The passive force $\boldsymbol{F}_{p}$ acts in direction of the cutting tool, and the feed force $\boldsymbol{F}_{f}$ in opposite direction of the feed. These forces are shown in Fig. 12.

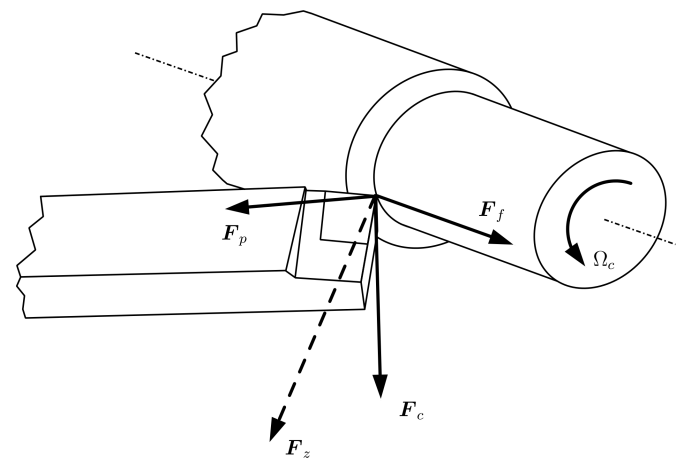

Fig. 12: Cutting forces in turning operation

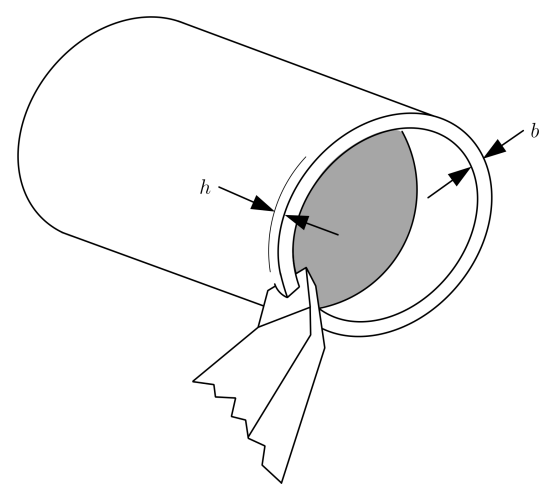

Fig. 13: Dimensions in orthogonal cutting

In this work we focus on computing the cutting force $\boldsymbol{F}_{c}$ with the PFEM and we want to compare the results to a turning process with an orthogonal cut as demonstrated in Fig. 13. Orthogonal cutting is 
characterised by orthogonal axes of cutting tool and workpiece. Derived in [23] and later mentioned in textbooks from manufacturing science as $[22,21]$, the formula

$$
F_{c}=\left|\boldsymbol{F}_{c}\right|=b h k_{c}
$$

can be applied to estimate the cutting force. Since the cutting force in this work is computed in a plane strain setting, the cutting thickness is set to $b=1 \mathrm{~mm}$. In Eq. (32) $h$ is the undeformed chip thickness and $k_{c}$ is defined as

$$
k_{c}=\frac{k_{c 1.1}}{h^{z}} k_{\lambda}\left(\lambda_{1}, \ldots, \lambda_{n}\right) .
$$

The constants $k_{c 1.1}$, and $z$ are material specific parameters which can be found in literature as [22] and $k_{\lambda}$ is an influence factor, which can be used to account for friction, shape of the cutting tool, etc.. However, in this work we set $k_{\lambda}=1$ to reduce the number of parameters. For the comparison between the results of the Kienzle formula in Eq. (32) and the cutting force from the PFEM simulations, we focus on the material S $235 \mathrm{JR}$. Then the parameters in Eq. (33) follow to $k_{c 1.1}=1780 \mathrm{MPa}$ and $z=0.17$. The undeformed chip thickness is set to $h=0.5 \mathrm{~mm}$ and the parameters used to describe the elastoplastic material in the PFEM simulations are $E=210,000 \mathrm{MPa}, \nu=0.3, \sigma_{i y}=235 \mathrm{MPa}$, $k=2500 \mathrm{MPa}, m=0.8$, and $\alpha=0.8$. Figure 14 shows the cutting force from the PFEM simulation and the estimate from Eq. (32).

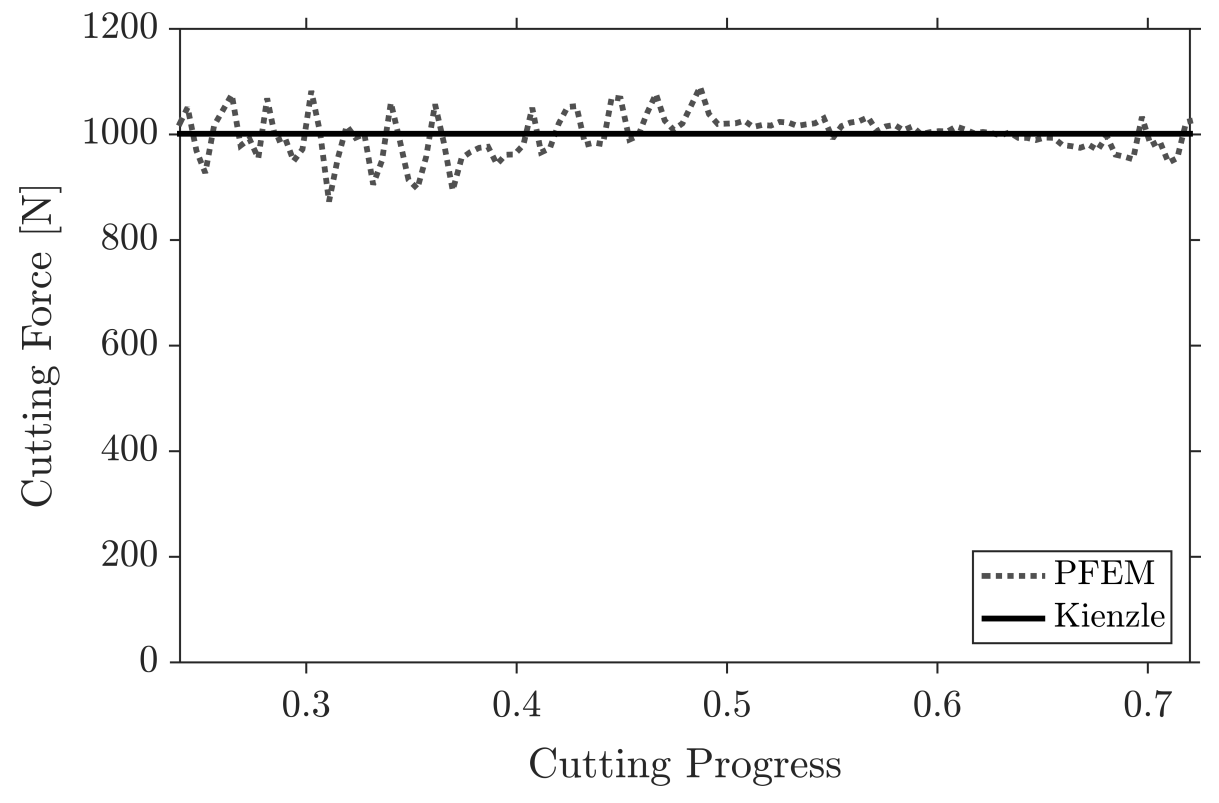

Fig. 14: Comparison to analytical benchmark

As can be seen in Fig. 14 the PFEM simulation is capable to reproduce the analytical result from Eq. (32).

\section{Conclusion}

The PFEM combines the benefits of continuum based and discrete modelling techniques and therefore provides a convenient tool for cutting simulations. In this work an outline of the method is provided and the finite element implementation is explained. It is shown that the $\alpha$-shape method influences the structural integrity of the material and that the parameter $\alpha$ can be interpreted as a maximum stretch of a boundary segment. A tensile test demonstrates this effect and $\alpha$ can be identified as a critical axial strain for the specimen. In the final section cutting simulations are presented. The formation of chips is demonstrated, as well as the distribution of stress and the plastic deformation during the cutting 
process. A parameter study illustrates that $\alpha$ influences the cutting force and the chip formation. Small values of $\alpha$ can be associated with rather brittle material, where ductile deformations can be observed for an increasing $\alpha$. Finally, cutting forces calculated with the PFEM and an analytical approach known from literature are compared.

\section{Acknowledgement}

This research was funded by the German Research Foundation (DFG) within the IRTG 2057 Physical Modeling for Virtual Manufacturing Systems and Processes.

\section{References}

[1] E. Oñate, S. R. Idelsohn, F. Del Pin, R. Aubry, The Particle Finite Element Method. An Overview, International Journal of Computational Methods, 1, 267-307 (2004)

[2] S. R. Idelsohn, E. Oñate, F. Del Pin, N. Calvo, Fluid-Structure Interaction Using the Particle Finite Element Method

[3] R. Holtermann, S. Schumann, A. Menzel, D. Biermann, Modelling, simulation and experimental investigation of chip formation in internal traverse grinding, Prod. Eng. Res. Devel., vol 7, 251263, doi:10.1007/s11740-013-0449-3 (2013)

[4] E. Oñate, A. Franci, J. M. Carbonell, A particle finite element method for analysis of industrial forming processes, Comput Mech, vol 54, 85-107, doi:10.1007/s00466-014-1016-2 (2014)

[5] M. Sabel, C. Sator, T. Zohdi, R. Müller, Application of the Particle Finite Element Method in Machining Simulation - Discussion of the Alpha-Shape Method in the Context of Strength of Materials, ASME. J. Comput. Inf. Sci. Eng., vol 17, doi:10.1115/1.4034434 (2016)

[6] M. Sabel, C. Sator, R. Müller, Simulation of Cutting Processes by the Particle Finite Element Method, GAMM Mitteilungen, (accepted 2016)

[7] J. R. Shewchuk, Triangle: Engineering a 2D Quality Mesh Generator and Delaunay Triangulator, Applied Computational Geometry: Towards Geometric Engineering, 1148, 203-222 (1996)

[8] R. L. Taylor, FEAP - A Finite Element Analysis Program: User Manual, 596, Department of Civil and Environmental Engineering, University of California, Berkley (2009)

[9] H. Edelsbrunner, E. P. Mücke, Three-Dimensional Alpha Shapes, ACM Transactions on Graphics, 13, 43-72 (1994)

[10] K. Fischer, Introduction to Alpha Shapes, Department of Information and Computing Sciences, Faculty of Science, Utrecht University, 17 (2000)

[11] G. Holzapfel, Nonlinear Solid Mechanics, 455, John Wiley \& Sons, Graz (2000)

[12] A. J. M. Spencer, Continuum Mechanics, 183, Dover Publications, Nottingham (2004)

[13] R. Greve, Kontinuumsmechanik, 495, Springer Verlag, Darmstadt (2003)

[14] A. Cuitiño, M. Ortiz, A material independent Method for extending Stress Update Algorithms from small Strain Plasticity to finite Plasticity with multiplicative Kinematics, Engineering Computations, 9, 437-451 (1992) 
[15] J. C. Simo, Algorithms for static and dynamic multiplicative plasticity that preserve the classical return mapping schemes of the infinitesimal theory, Comput Methods Appl Mech Eng, vol 99, 61-112 (1992)

[16] F. J. Montáns, K. J. Bathe, On the stress integration in large strain elasto-plasticity, Computational Fluid and Solid Mechanics, 494-497, DOI: 10.1016/B978-008044046-0.50122-6 (2003)

[17] K. J. Bathe, Finite Element Procedures, 1037, Prentice Hall, Cambridge (1996)

[18] J. C. Simo, T. J. R. Hughes, Computational Inelasticity, 392, Springer (1991)

[19] E. d. S. Neto, D. Peric, D. Owens, Computational methods for plasticity : theory and applications, 791,Wiley (2008)

[20] E. H. Lee, Elastic-Plastic Deformation at Finite Strains, Journal of Applied Mechanics, 36, 1-6 (1969)

[21] B. Denkena, H. K. Tönshoff, Spanen - Grundlagen, 426, Springer (2011)

[22] A. Böge, Formeln und Tabellen Maschinenbau, 390, Springer (2012)

[23] O. Kienzle, Die Bestimmung von Kräften und Leistungen an spanenden Werkzeugen und Werkzeugmaschinen, Z. VDI, Bd. 94, (1952) 